\title{
Novel embryonic stem cells expressing tdKaede protein photoconvertible from green to red fluorescence
}

\author{
YOKO SHIGEMATSU ${ }^{*}$, NAOKI YOSHIDA $^{2 *}$, YOSHIHIRO MIWA $^{2}$, ATSUSHI MIZOBUTI $^{1}$, \\ YUKO SUZUKI $^{1}$, YOKO TANIMOTO ${ }^{1}$, SATORU TAKAHASHI $^{1}$, SATOSHI KUNITA ${ }^{1}$, \\ FUMIHIRO SUGIYAMA $^{1}$ and KEN-ICHI YAGAMI ${ }^{1}$ \\ ${ }^{1}$ Laboratory Animal Resource Center and ${ }^{2}$ Department of Molecular Pharmacology, Graduate School of \\ Comprehensive Human Sciences, University of Tsukuba, Tsukuba, Ibaraki 305-8575, Japan
}

Received April 13, 2007; Accepted May 28, 2007

\begin{abstract}
Kaede protein is a photoconvertible tracer that emits green fluorescence after synthesis, which changes to stable red fluorescence upon irradiation with violet or UV illumination. This color-change characteristic is a very effective means of optically marking living cells of interest. We established novel embryonic stem (ES) cell lines, B6KED-1 and -2, from C57BL/6J transgenic mouse blastocysts ubiquitously expressing tandem dimeric Kaede (tdKaede) protein. Undifferentiated B6KED-1 and -2 cells showed bright green fluorescence and mRNAs of pluripotent marker genes. Photoconversion of tdKaede protein in undifferentiated and differentiated B6KED cells in vitro occurred upon short-term UV irradiation. B6KED cells completely generated ES cell-derived females on transfer into tetraploid blastomeres. All organs showed strong green emission in the females derived completely from B6KED cells. These novel ES cell lines ubiquitously expressing photoconvertible Kaede protein, B6KED-1 and -2, are useful for basic research in developmental biology and regenerative medicine.
\end{abstract}

\section{Introduction}

Embryonic stem (ES) cells were established from 3.5-day mouse blastocyst inner cell mass $(1,2)$. These cells can proliferate extensively and identically in the undifferentiated condition and can differentiate into a variety of cell types of

Correspondence to: Dr Fumihiro Sugiyama, Laboratory Animal Resource Center, University of Tsukuba, 1-1-1 Tennodai, Tsukuba, Ibaraki 305-8575, Japan

E-mail: bunbun@md.tsukuba.ac.jp

${ }^{*}$ Contributed equally

Key words: embryonic stem cells, mouse, Kaede, photoconversion, ubiquitous expression all three germ layers: ectoderm, mesoderm, and endoderm. Furthermore, due to their ability to differentiate into germ cells, ES cells have been used as a powerful vehicle for transgenesis. Gene-targeted mice derived from ES cells treated by homologous recombination have facilitated the genetic dissection of developmental mechanisms in vivo. This knowledge is useful for the development of advanced methods of in vitro differentiation using ES cells. A previous study in which human ES cells were established from growing blastocytes indicated the necessity for a radical new approach to using ES cells in the treatment of chronic and degenerative disease states. However, human ES cells are more difficult to examine in vivo than mouse ES cells.

Previously, we reported the establishment of a mouse ES cell line, named B6G-2, derived from C57BL/6 mice ubiquitously expressing enhanced green fluorescent protein (EGFP). EGFP and similar fluorescent proteins are useful for visualizing the locations of differentiated ES cells in living mice. Ando et al (3) cloned a cDNA encoding a fluorescent protein named Kaede from the red open brain coral Trachyphyllia geoffroyi, which shows a pronounced photoinduced spectral change. Similar to EGFP, Kaede protein emits bright green fluorescence after synthesis, but changes efficiently to a bright and stable red fluorescence when irradiated with UV or violet light. They showed that the property of photoconversion is useful for marking living single cells of interest in dense cultures.

In organ formation, cell fate is regulated by a combination of cell-specific genes that control intracellular events and responses to the environment, such as cell-cell and extracellular matrix-mediated interactions (4). To understand the mechanisms of differentiation through ES cells in detail, Kaede proteins may have the advantage of statically tracing the positions of living cells of interest. Previously, we (Miwa Y, unpublished data) generated a transgenic mouse line carrying tandem dimeric Kaede (tdKaede) genes under the regulation of the $\beta$-actin promoter fused with cytomegalovirus minimum enhancer, CMV (5). In the present study, we established novel ES cell lines from growing blastocysts of C57BL/6JTgN(act-tdKaede)UTR, nicknamed C57BL/6J-tdKaede, to obtain ES cells useful for optical marking in a variety of cells. 


\section{Materials and methods}

Animals. C57BL/6J-TgN(act-tdKaede)UTR were generated at the Laboratory Animal Resource Center, University of Tsukuba. They were kept in plastic cages (4-5 per cage) under pathogen-free conditions (sentinel mice were periodically examined throughout the study) in a room at $23.5 \pm 2.5^{\circ} \mathrm{C}$ and $52.5 \pm 12.5 \%$ relative humidity, and under a 14-h light:10-h dark cycle. Mice had free access to a commercial chow (MF; Oriental Co., Ltd., Tokyo, Japan) and autoclaved water. All study protocols were approved by the University Animal Experimental Committee at the University of Tsukuba.

Embryos. To conduct in vitro fertilization, sperm were collected from the epididymides of heterogeneous C57BL/6JtdKaede transgenic mice. Unfertilized eggs were collected from C57BL/6J mice treated with pregnant mare serum gonadotropin and human chorionic gonadotropin. In vitro fertilization was performed as described previously (6). The fertilized embryos were frozen at the two-cell stage until resumption of embryo culture.

Establishment of ES cells. Frozen-thawed two-cell embryos were cultured to the blastocyst stage in KSOM medium. Thirty-six blastocysts, from which the zonae pellucidae had been removed by brief exposure to acidic Tyrode's solution, were placed on a feeder layer of mitomycin C-inactivated confluent embryonic fibroblasts in 4-well dishes. For culture of ES cells, Dulbecco's modified Eagle's medium was supplemented with $1 \%$ fetal calf serum, 15\% KSR (Invitrogen, San Diego, CA), 0.1 mM 2-mercaptoethanol, 1000 units $/ \mathrm{ml}$ LIF (ESGRO), nonessential amino acids, and sodium pyruvate. A vigorous inner cell mass (ICM) appeared within the attached blastocysts by the 5th day of cultivation in the above medium. To expand the next generation, the ICM was extracted with a micropipette, dissociated to single cells with trypsin, and seeded onto a new feeder layer. Subculture was performed at intervals of 2 days after passage 4 . ES-like colonies were never allowed to become larger than $400 \mu \mathrm{m}$ in diameter. The putative ES cells were frozen at a concentration of $2 \times 10^{6}$ cells per vial on passage 7 . Cultures were maintained in a humidified incubator at $37^{\circ} \mathrm{C}$, under an atmosphere of $5 \% \mathrm{CO}_{2}$ and $95 \%$ air. We used B6KED-1 and -2 cells after passage 8 to examine the potential for pluripotency both in vitro and in vivo.

Alkaline phosphatase activity. The pluripotent phenotype was assessed using an alkaline phosphatase staining kit (Sigma, St. Louis, MO). The putative ES cells on the feeder layer were fixed with $4 \%$ paraformaldehyde solution, dried, and stained for alkaline phosphatase activity in the cytoplasm using the kit in accordance with the manufacturer's specifications.

RT-PCR analysis. The expression of pluripotent marker genes was determined by reverse transcription-PCR (RTPCR). Total RNA was isolated using an Isogen RNA extraction kit (Nippongene, Tokyo, Japan). First-strand cDNA was prepared with oligo(dT) using the SuperScript II firststrand synthesis system (Invitrogen). cDNAs were amplified by PCR for 27 cycles. The following specific primers were used for analyses of Oct3/4 and Nanog gene expression: Oct3/4 (AACCTGGCTAAGCTTCCAAG and GAGTAG AGTGTGGTGAAGTG) generating a fragment of 894 bp and Nanog (CAGTTTGCCTAGTTCTGAGG and CCAAA TCACTGGCAGAGAAG) generating a fragment of $788 \mathrm{bp}$. For analyses of $\beta$-actin gene expression as an internal standard, the following specific primers were used: $\beta$-actin (GCTGTA TTCCCCTCCATCGTG and GGATGGCTACGTACAT GGCTG) generating a fragment of $325 \mathrm{bp}$. PCR products were electrophoresed through $2 \%$ agarose gels and stained with ethidium bromide.

In vitro differentiation of ES cells. The capability of the putative ES cells to differentiate in vitro was examined using the methods of Strubing et al (7). To induce the formation of embryoid bodies, aliquots of $4 \times 10^{2}$ ES cells were placed in drops of $20 \mu \mathrm{l}$ of DMEM containing $20 \% \mathrm{FCS}, 10^{-7}$ all-trans retinoic acid (Sigma), $2 \mathrm{mM}$ glutamine (Invitrogen), nonessential amino acids, and $50 \mu \mathrm{M} 2$-mercaptoethanol on nontissue culture-grade plates. The cells were cultured according to the hanging drop method for 2 days to form embryo-like aggregates. The embryoid bodies were then collected, washed, and plated on dishes coated with gelatin in the above medium without all-trans retinoic acid to allow them to attach and differentiate.

ES cell-tetraploid embryo complementation. We prepared two-cell embryos from ICR females that had been superovulated and mated naturally. Electrofusion was performed using a Model LF101, Electro Cell Fusion (NepaGene, Ichikawa, Japan). Two-cell embryos were placed between the electrodes in $0.3 \mathrm{M}$ mannitol solution containing $0.03 \%$ bovine serum albumin. The embryo orientation was provided by a $1 \mathrm{MHz}$ AC field (10 V, $30 \mathrm{sec})$, and fusion was carried out at $15 \mu \mathrm{sec}$ (pulse width) DC field $(150 \mathrm{~V}, 1 \mathrm{sec})$. The tetraploid embryos were cultured to the 4-cell stage in KSOM. To produce chimeric embryos, the tetraploid blastomeres with the zonae pellucidae removed were aggregated with B6KED cells according to the sandwich method of Wood et al (8). The chimeric blastomeres were developed to the blastocyst stage in vitro and transferred to the uteri of pseudopregnant females.

\section{Results}

Establishment of a clonal cell line derived from C57BL/6tdKaede blastocysts. Previously, we obtained ES cell lines from blastocysts developed through in vitro fertilization, cryopreservation, and cultivation under appropriate conditions (9). In the present study, oocytes of C57BL/6J mice fertilized in vitro with the sperm of C57BL/6J-tdKaede mice were prepared as the initial source to establish ES cell lines. After cryopreservation at the 2-cell stage, thawed embryos were cultured to the blastocyst stage and then placed on an embryonic fibroblast feeder layer. Two clonal cell lines, named B6KED-1 and B6KED-2, were established from 36 zona-free blastocysts and were examined morphologically. As reported previously, in mouse ES cells $(1-3,9)$, the clonal cells of B6KED-1 and -2 adhered tightly to each 

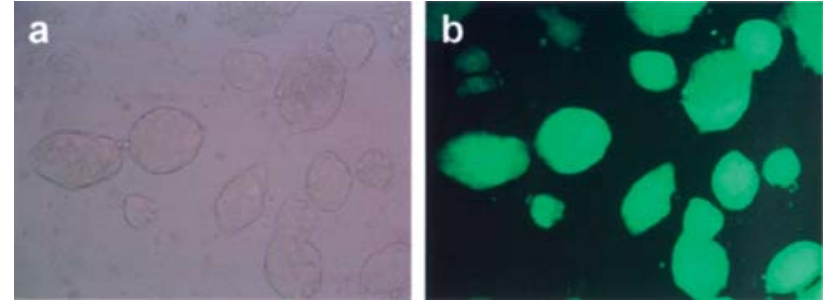

Figure 1. Morphology of B6KED cells. (a) B6KED-2 cells formed ES celllike colonies. (b) Strong green emission was observed in B6KED-2 colonies under illumination with blue light.

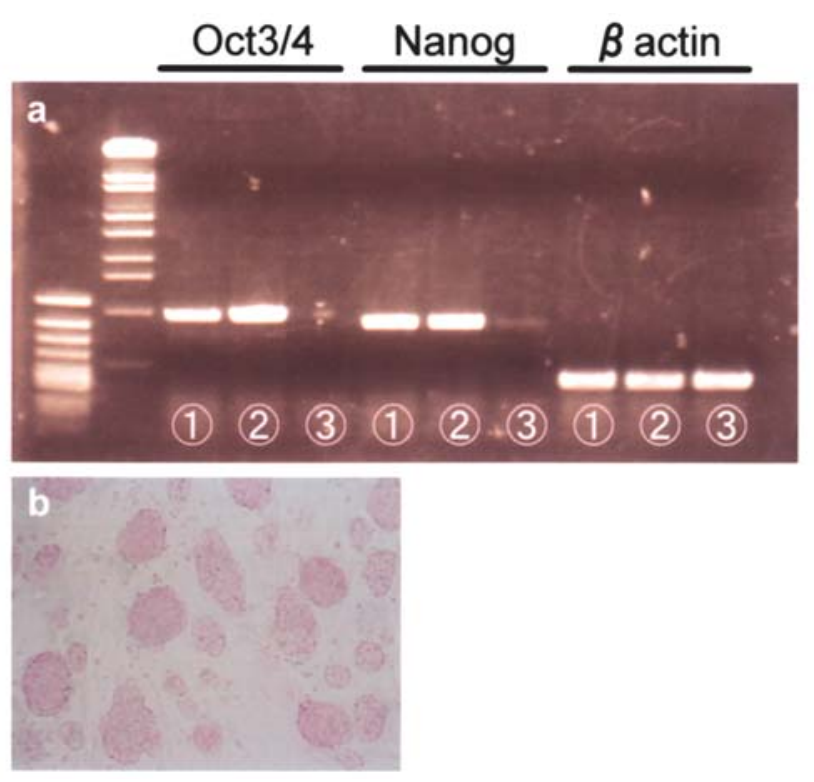

Figure 2. Analyses of pluripotent markers in B6KED cells. (a) Oct3/4 and Nanog expression was observed in B6KED-1 and -2. Detection of $B$-actin mRNA was carried out as a control for RT-PCR analysis. The encircled number 1, 2, and 3 indicates B6KED-1, -2, and feeder cells, respectively. (b) Alkaline phosphatase activity in B6KED-2 cells was examined biochemically. Colonies of B6KED-2 cells developed violet color, indicating high alkaline phosphatase activity.

other, making it impossible to visualize individual cells in the colonies, which were readily distinguishable from the feeder layers (Fig. 1a). When illuminated with blue-spectrum light at wavelengths of 460-500 $\mathrm{nm}$, strong green fluorescence was observed in ES-like colonies of B6KED-1 and -2, but not in the feeder layer cells (Fig. 1b).

Expression of pluripotent marker genes in B6KED cells. To determine whether established clonal cells express marker genes controlling pluripotency, alkaline phosphatase activity and mRNAs of Oct3/4 and Nanog were examined in expanded B6KED-1 and - 2 cells. Alkaline phosphatase is expressed in primordial germ cells and teratocarcinoma cells (10), and its activity is generally used as a marker of pluripotent cells. Oct3/4, the known POU domain, class 5, transcription factor 1 , designated as Pou5f1, is essential for the identity of the pluripotential founder cell population in mouse embryos (11). Nanog is a divergent homeodomain protein that directs propagation of undifferentiated ES cells $(12,13)$. mRNAs of
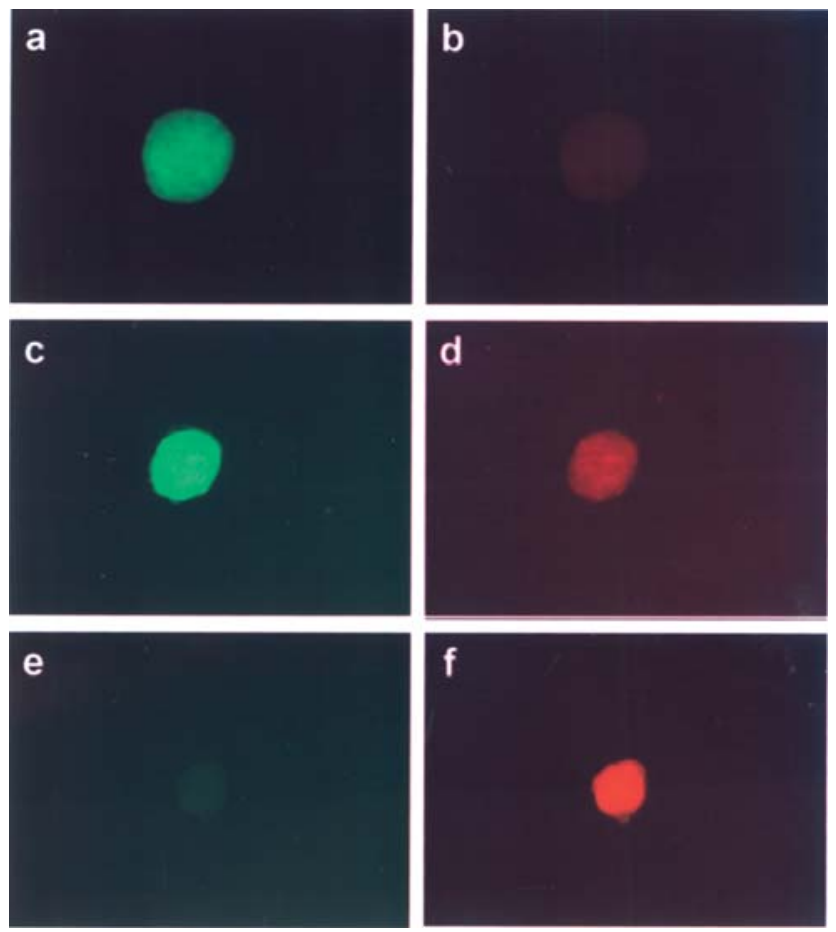

Figure 3. Photoconversion of B6KED colonies. Each colony was irradiated with UV light of an appropriate wavelength for $10 \mathrm{sec}(\mathrm{a}, \mathrm{b}), 30 \mathrm{sec}(\mathrm{c}, \mathrm{d})$, or 10 min $(e, f)$. After UV irradiation, the photoconversion of colonies was observed under appropriate blue $(\mathrm{a}, \mathrm{c}, \mathrm{e})$ and green $(\mathrm{b}, \mathrm{d}, \mathrm{f})$ light.

Oct3/4 and Nanog were detected in B6KED-1 and -2 cells, which formed typical ES-like colonies in appropriate medium containing leukemia inhibitory factor, but not in feeder cells (Fig. 2a). As shown in Fig. 2b, alkaline phosphatase activity was observed in colonies of B6KED-1 and -2 cells on top of feeder layers with no activity. These results suggest that B6KED-1 and -2 cells retain the property of vigorous self-renewal in the undifferentiated state.

Photoconversion of tdKaede proteins in undifferentiated B6KED cells. To investigate whether tdKaede protein undergo photoconversion in pluripotent ES cells, we irradiated clonal colonies consisting of B6KED cells on the feeder layer with a UV lamp through a standard 340- to 380-nm UV filter for $10 \mathrm{sec}, 30 \mathrm{sec}$, and $10 \mathrm{~min}$. After UV irradiation, ES colonies were observed through a standard FITC filter (excitation filter $480 / 40 \mathrm{~nm}$, dichroic $505 \mathrm{~nm}$, absorption filter 527/30 nm) and a standard rhodamine filter (excitation filter 546/12 nm, dichroic $565 \mathrm{~nm}$, absorption filter 600/40 nm) for green and red fluorescence of tdKaede protein, respectively. Upon UV irradiation for $10 \mathrm{sec}$, B6KED cells showed generation of photoconverted Kaede protein red fluorescence (Fig. 3a and b). Photoinduced red colonies were clearly visible upon UV irradiation for $30 \mathrm{sec}$, although these colonies also retained bright green emission (Fig. 3c and d). After $10 \mathrm{~min}$ of UV illumination, Kaede protein showed complete photoconversion from green to red fluorescence (Fig. 3e and f).

In vitro differentiation of B6KED cells. In culture without LIF, murine ES cells generally differentiated into a variety of cell types after embryoid body formation. To investigate the 

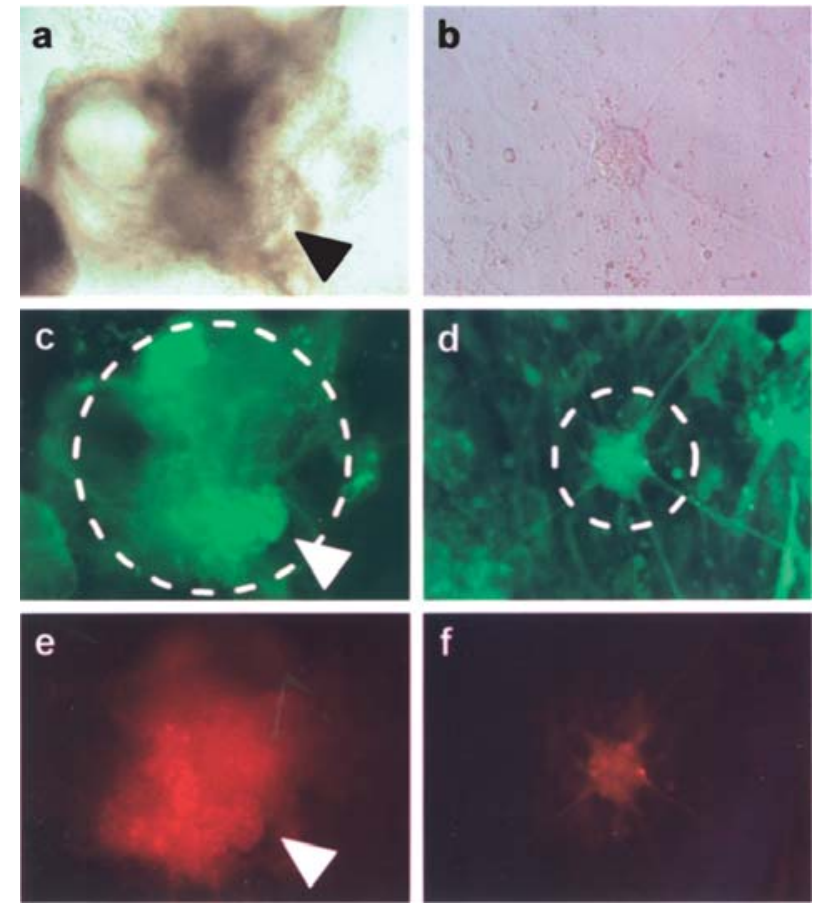

Figure 4. In vitro differentiation of B6KED cells and photoconversion of tdKaede protein in differentiated B6KED cells. The contracting mass (arrowheads in a) and neuronal-like cells with long processes were generated from proliferated embryoid bodies after attachment on gelatincoated dishes. All derivates with green fluorescence $(c, d)$ showed photoinduced color change from green to red $(e, f)$ when irradiated with UV in the area marked by a dotted circle.

Table I. Production of complete chimera mice derived from B6KED cells and tetraploid blastomeres.

\begin{tabular}{lccc}
\hline Clones & $\begin{array}{c}\text { No. of chimera } \\
\text { embryos transferred }\end{array}$ & $\begin{array}{c}\text { No. of } \\
\text { newborns }\end{array}$ & Males:females \\
\hline B6KED-1 & 100 & 10 & $0: 10$ \\
B6KED-2 & 100 & 26 & $0: 26$ \\
Total & 200 & 36 & $0: 36$ \\
\hline
\end{tabular}

in vitro differentiation of B6KED cells, embryo bodies composed of B6KED cells were formed in hanging drops with retinoic acid and without LIF. The B6KED cell aggregates were spread out on gelatin-treated dishes and cultured in differential medium without retinoic acid and LIF. Attached cells propagated progressively, aggregating in the center of colonies and expanding to form a monolayer around the colonies. After 7 days in the differential medium, B6KED-2 cells began rhythmic contraction in a relatively cell-dense area (Fig. 4a, c and e). The appearance of the contractile structure suggests that B6KED-2 cells differentiated to cardiac smooth muscle cells. At least 14 days after plating, B6KED-2 cells formed neuronal cells with several long processes and neural rosettes in areas of low cell density (Fig. 4b, d and f). The neuronal and smooth muscle cells generated from the undifferentiated B6KED-2 cells showed bright green fluorescence when illuminated with blue light
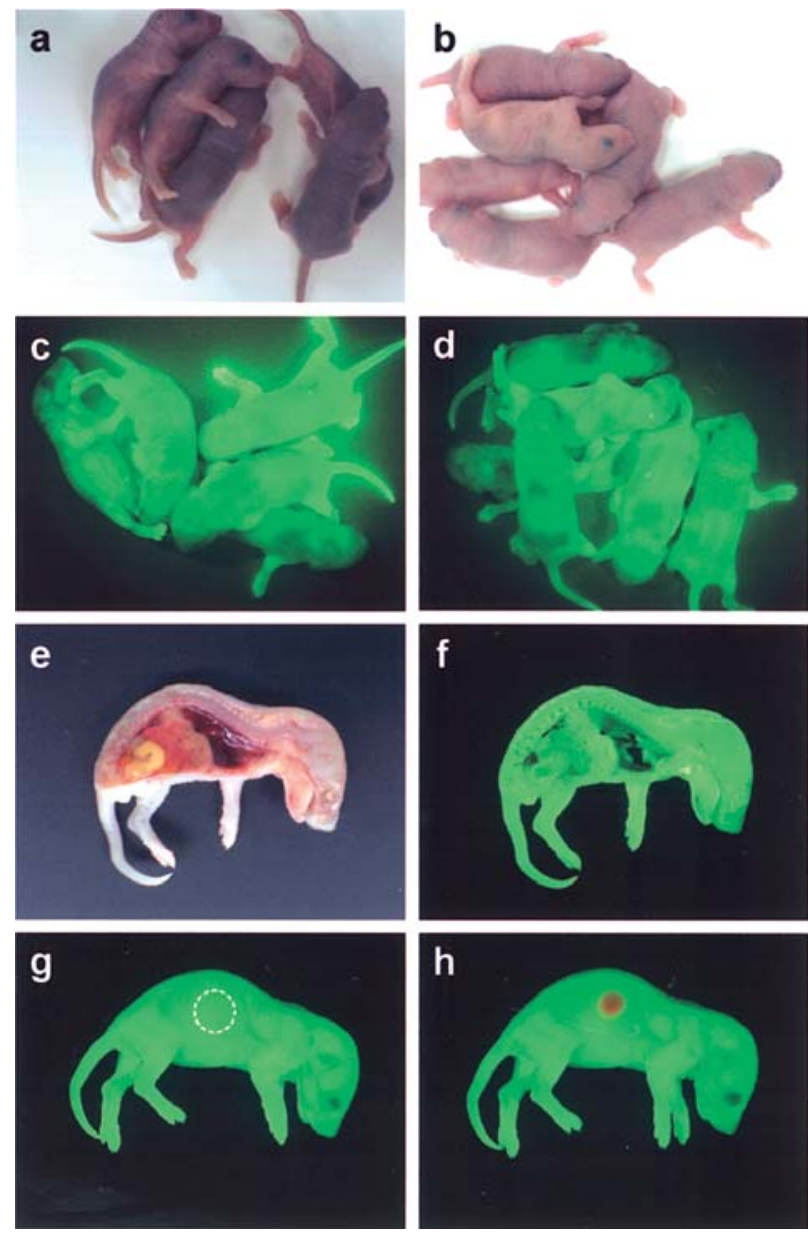

Figure 5. B6KED cell-tetraploid mice. Newborn females were produced from B6KED-1 (a,c) and -2 (b,d,e,f,g,h) cells by tetraploid embryo complementation. Green fluorescence was observed ubiquitously in all neonates derived from B6KED cells (c,d). A B6KED-2 cell-tetraploid mouse was fixed with $4 \%$ paraformaldehyde and sliced sagittally (e). All organs showed strong green emission in the midsagittal sections (f). When irradiated with UV in the area marked by a dotted circle $(\mathrm{g})$, the skin of living mice showed a dramatic change in color from green to red (h).

(Fig. 4c and d), but not red fluorescence when illuminated with green light (data not shown). After UV irradiation for $30 \mathrm{sec}$, tdKaede protein was converted to red fluorescence in differentiated B6KED-1 cells (Fig. 4e and f). These findings, which were confirmed in B6KED-1 cells (data not shown), indicate that B6KED-1 and -2 cells are pluripotent embryonic stem cells.

In vivo differentiation of totipotent B6KED cells. Finally, we investigated whether B6KED-1 and -2 cells have great potential for differentiating into all cell lineages in vivo. Pluripotent B6KED ES cells were aggregated between two tetraploid blastomeres of ICR mice to generate ES celltetraploid mice derived completely from the ES cells. We surgically transferred 100 chimeric embryos into the uteri of pseudopregnant female mice for each ES cell line. Despite ES cell-tetraploid embryo complementation, we obtained a large number of newborn mice through normal delivery. Almost all neonates were alive and showed no abnormalities after weaning. All B6KED-1 and -2 cell tetraploid mice were female. These results are summarized in Table I. The 
complete chimeric neonates derived from B6KED-1 (Fig. 5a and c) and -2 (Fig. 5b and d) cells showed bright green fluorescence in their skin (excitation filter $470 / 40 \mathrm{~nm}$, absorption filter $510 \mathrm{~nm} /$ long pass). In midsagittal sections of neonates derived from B6KED-2 cell-tetraploid embryos, a bright field was seen in a variety of organs, including the brain, spinal cord, thymus, lung, heart, liver, and intestine (Fig. 5e). Strong green signals were detected in all tissues except a clot of blood in the cardiac ventricle (Fig. 5f). To determine the photoconversion of tdKaede protein in vivo, the abdominal skin of B6KED-2 cell-tetraploid neonates was irradiated for 1 min with UV (Fig. 5g); tdKaede protein showed a dramatic change from green to red fluorescence in the developing mice (Fig. 5h).

\section{Discussion}

Green fluorescent protein and its homologs can be used for efficiently visualizing living cells (14). Recently, we developed a unique C57BL/6-ES cell line, B6G-2, ubiquitously expressing EGFP as a useful resource for basic research in regenerative medicine (9). The foreign tdKaede mRNA in B6KED cells was also expressed under the control of the ß-actin promoter fused with CMV (5), suggesting ubiquitous expression in the developing mouse. Indeed, green fluorescence was observed throughout development in B6KED cell-tetraploid mice. Furthermore, B6KED cells have the genetic background of $\mathrm{C} 57 \mathrm{BL} / 6 \mathrm{~J}$, a common inbred mouse strain. Therefore, B6KED cells carrying the tdKaede gene permit chimera experiments in allogeneic transplantation as well as B6G-2 cells, but B6G-2 cells cannot be labeled to trace single or multiple cells in a dense cell population. The most distinctive feature of B6KED cells is that they provide a simple means of marking living cells. Sato et al (15) reported that red fluorescence emitted from photoconverted Kaede was traced selectively to neurons during dendrite development for 3 days in zebrafish, indicating that Kaede protein is a useful tool for investigating the construction of neural networks in vivo. In the present study, we used conventional wide-field microscopy for UV irradiation of a region of interest. Although this study demonstrated the color-change properties of ES cells and their derivates in vitro and in vivo, long-term and wide-ranging exposure to UV irradiation may affect the cell nuclei. Further studies are required to investigate the effects of irradiation with violet light at longer wavelengths than that used in the present study and/or use of laser scanning confocal microscopy or pulse laser for irradiation of the cytosolic compartment with light at the violet wavelength.

ES cells with the C57BL/6 genetic background are beneficial to the generation of knockout mice, which are unnecessary to backcross to C57BL/6. Several groups reported the establishment of ES cells with the C57BL/6 genetic background (9,16-18). Most ES cell lines were derived from male embryos, indicating generation of male gametes. B6KED-1 and -2 cells had the genetic background of $\mathrm{C} 57 \mathrm{BL} / 6 \mathrm{~J}$ and further contributed to the generation of female gametes (Sugiyama F, unpublished data). To the best of our knowledge, this is the first report of the establishment of available female ES cells with the C57BL/6 genetic background. Unlike male ES cells, female ES cells may be useful for studies of differentiation into female reproductive tissues and oocytes. Female ES cells rarely occur by Y chromosome loss in male ES cells. Although X0 female mice are viable and fertile (19), having only a single X chromosome may be disadvantageous to developing mice because of the lack of redundancy in aberrant DNA methylation. Although there is no observation of karyotype in the present study, B6KED cells showed the highest performance for production of ES cell-tetraploid mice as compared with our other experiments using the B6G-2 cell line (9), other C57BL/6J-ES cell lines, and the E14 cell line derived from the 129 mouse strain (Takahashi S, unpublished data). Therefore, B6KED cells have retained their potential to differentiate into cells of multiple lineages.

We established a novel ES cell line named B6KED from blastocysts of C57BL/6-tdKaede mice. ES cell lines showing ubiquitous expression of tdKaede protein, which shows photoconversion from green to red fluorescence upon UV irradiation, will be useful tools in developmental biology and transplantation medicine.

\section{Acknowledgements}

This study was supported by grants from the Ministry of Education, Science, Sport, and Culture of Japan (F.S. and K.Y.). The authors thank Keiko Furuya, Noriko Kajiwara (University of Tsukuba), and Hideki Nishikawa (Keyence Co., Osaka) for their excellent technical support.

\section{References}

1. Martin G: Isolation of a pluripotent cell line from early mouse embryos cultured in medium conditioned by teratocarcinoma cells. Proc Natl Acad Sci USA 78: 7634-7638, 1981.

2. Evans MJ and Kaufman MH: Establishment in culture of pluripotential stem cells from mouse embryos. Nature 292: 154-156, 1981.

3. Ando R, Hama H, Yamamoto-Hino M, Mizuno $\mathrm{H}$ and Miyawaki A: An optical marker based on the UV-induced green-to-red photoconversion of a fluorescent protein. Proc Natl Acad Sci USA 99: 12651-1266, 2002.

4. Linask KK, Manisastry S and Han M: Cross talk between cellcell and cell-matrix adhesion signaling pathways during heart organogenesis: implications for cardiac birth defects. Microsc Microanal 11: 200-208, 2005.

5. Niwa N, Yamamura K and Miyazaki J: Efficient selection for high-expression transfectants with a novel eukaryotic vector. Gene 108: 193-199, 1991.

6. Sugiyama F, Kajiwara N, Hayashi S, Sugiyama Y and Yagami K: Development of mouse oocytes superovulated at different ages. Lab Anim Sci 42: 297-298, 1992.

7. Strubing C, Ahnert-Hilger G, Shan J, Wiedenmann B, Hesheler J and Wobus AM: Differentiation of pluripotent embryonic stem cells into the neuronal lineage in vitro gives rise to mature inhibitory and excitatory neurons. Mech Dev 53: 275-287, 1995.

8. Wood SA, Allen ND, Rossant J, Auerbach A and Nagy A: Noninjection methods for the production of embryonic stem cellembryo chimeras. Nature 365: 87-89, 1993.

9. Shimizukawa R, Sakata A, Hirose M, Takahashi A, Iseki H, Liu Y, Kunita S, Sugiyama F and Yagami K: Establishment of a new embryonic stem cell line derived from C57BL/6 mouse expressing EGFP ubiquitously. Genesis 42: 47-52, 2005.

10. Strickland S, Smith KK and Marotti KR: Hormonal induction of differentiation in teratocarcinoma stem cells: generation of parietal endoderm by retinoic acid and dibutyryl cAMP. Cell 21: 347-355, 1980.

11. Nichols J, Zevnik B, Anastassiadis K, Niwa H, Klewe-Nebenius D, Chambers I, Scholer $\mathrm{H}$ and Smith A: Formation of pluri-potent stem cells in the mammalian embryo depends on the POU transcription factor Oct4. Cell 95: 379-391, 1998. 
12. Chambers I, Colby D, Robertson M, Nichols J, Lee S, Tweedie S and Smith A: Functional expression cloning of Nanog, a pluripotency sustaining factor in embryonic stem cells. Cell 30: 113: 643-655, 2003.

13. Mitsui K, Tokuzawa Y, Itoh H, Segawa K, Murakami M, Takahashi K, Maruyama M, Maeda M and Yamanaka S: The homeoprotein Nanog is required for maintenance of pluripotency in mouse epiblast and ES cells. Cell 113: 631-642, 2003.

14. Shaner NC, Steinbach PA and Tsien RY: A guide to choosing fluorescent proteins. Nat Methods 12: 905-909, 2005.

15. Sato T, Takahoko M and Okamoto H: HuC:Kaede, a useful tool to label neural morphologies in networks in vivo. Genesis 44: 136-142, 2006.
16. Ledrmann P and Burki K: Establishment of a germ-line competent C57BL/6 embryonic stem cell line. Exp Cell Res 197: 254-258, 1991

17. Baharvand $\mathrm{H}$ and Matthaei KI: Culture condition difference for establishment of new emrbryonic stem cell lines from the C57BL/6 and BALB/c mouse strains. In Vitro Cell Dev Biol 40: 76-81, 2004.

18. Cheng J, Dutra A, Takesono A, Garrett-Beal L and Schwartzberg PL: Improved generation of C57BL/6J mouse embryonic stem cells in a defined serum-free media. Genesis 39: 100-104, 2004.

19. Cattanach BM: X0 mice. Genet Res 3: 487-490, 1962. 